\title{
BMJ Open Value of social network analysis for developing and evaluating complex healthcare interventions: a scoping review
}

\author{
Linda C Smit (D) , ${ }^{1}$ Jeroen Dikken, ${ }^{2}$ Marieke J Schuurmans, ${ }^{3}$ Niek J de Wit, ${ }^{4}$ \\ Nienke Bleijenberg ${ }^{1,5}$
}

To cite: Smit LC, Dikken J, Schuurmans MJ, et al. Value of social network analysis for developing and evaluating complex healthcare interventions: a scoping review. BMJ Open 2020;10:e039681. doi:10.1136/ bmjopen-2020-039681

- Prepublication history and additional material for this paper is available online. To view these files, please visit the journal online (http://dx.doi.org/10. 1136/bmjopen-2020-039681).

Received 22 April 2020 Revised 21 0ctober 2020 Accepted 26 0ctober 2020
Check for updates

\section{(c) Author(s) (or their} employer(s)) 2020. Re-use permitted under CC BY-NC. No commercial re-use. See rights and permissions. Published by BMJ.

For numbered affiliations see end of article.

Correspondence to

Linda C Smit; linda.smit@hu.nl

\section{ABSTRACT}

Objectives Most complex healthcare interventions target a network of healthcare professionals. Social network analysis (SNA) is a powerful technique to study how social relationships within a network are established and evolve. We identified in which phases of complex healthcare intervention research SNA is used and the value of SNA for developing and evaluating complex healthcare interventions.

Methods A scoping review was conducted using the Arksey and 0'Malley methodological framework. We included complex healthcare intervention studies using SNA to identify the study characteristics, level of complexity of the healthcare interventions, reported strengths and limitations, and reported implications of SNA. The Preferred Reporting Items for Systematic Reviews and Meta-Analyses Extension for Scoping Reviews 2018 was used to guide the reporting.

Results Among 2466 identified studies, 40 studies were selected for analysis. At first, the results showed that SNA seems underused in evaluating complex intervention research. Second, SNA was not used in the development phase of the included studies. Third, the reported implications in the evaluation and implementation phase reflect the value of SNA in addressing the implementation and population complexity. Fourth, pathway complexity and contextual complexity of the included interventions were unclear or unable to access. Fifth, the use of a mixed methods approach was reported as a strength, as the combination and integration of a quantitative and qualitative method clearly establishes the results. Conclusion SNA is a widely applicable method that can be used in different phases of complex intervention research. SNA can be of value to disentangle and address the level of complexity of complex healthcare interventions. Furthermore, the routine use of SNA within a mixed method approach could yield actionable insights that would be useful in the transactional context of complex interventions.

\section{INTRODUCTION}

The development and evaluation of interventions in healthcare are often considered to be complex. ${ }^{1}$ This complexity has been defined in various ways. ${ }^{23} \mathrm{~A}$ consolidated definition
Strengths and limitations of this study

- The Preferred Reporting Items for Systematic Reviews and Meta-Analyses Extension for Scoping Reviews 2018 was used to ensure the quality of reporting.

- Two researchers worked independently during the selection of studies, data charting and synthesis of the results. All authors checked and confirmed the synthesis of the results.

- The literature search was conducted in four scientific databases, which is more than sufficient to include the central and relevant research evidence regarding complex intervention studies in healthcare.

- There is no sharp boundary between simple and complex healthcare interventions; therefore, the level of complexity was unravelled of all included studies based on the iCAT_SR.

- This study did not critically appraise the included studies.

for complex interventions was therefore formulated by Guise $e t a l^{4}$.

All complex interventions have two common characteristics; they have multiple components (intervention complexity) and complicated/multiple causal pathways, feedback loops, synergies, and/ or mediators and moderators of effect (pathway complexity). In addition, they may also have one or more of the following three additional characteristics; target multiple participants, groups, or organisational levels (population complexity); require multifaceted adoption, uptake, or integration strategies (implementation complexity); or work in a dynamic multidimensional environment (contextual complexity). ${ }^{4}$

Additionally, interventions can be conceptualised as having 'core components', that is, the essential and indispensable elements 
of the intervention and an 'adaptable periphery', that is, adaptable elements, structures and systems related to the intervention and organisation into which it is being implemented. ${ }^{56}$

The effectiveness of complex interventions is critically influenced by their contexts. ${ }^{7-9}$ Context is often used synonymously with setting and environment and includes static (eg, the physical environment) and dynamic aspects in terms of professionals, relationships or networks. ${ }^{8}$ Because of the heterogeneity of the contexts in which complex interventions are embedded, there is still no adequate translation of how to accommodate to the context in good clinical practice. ${ }^{810}$ Furthermore, most complex interventions in healthcare research target a network of different (healthcare) professionals from multiple sectors and disciplines that is commonly driven by interactions. Such networks form the backbone of a system (eg, hospital, general practice) by directing the collective power of diverse individuals and groups to achieve mutually relevant goals and objectives. ${ }^{11}$ However, there is a lack of intervention studies exploring the underlying network structure and how this structure affects intervention outcomes as well as the contribution that different actors such as interventionists play in a network. ${ }^{12}$

Social network analysis (SNA) is a scientific method to study underlying network structures. SNA is a powerful technique that aims to characterise and study how social relationships within a network, for example, among persons, groups or organisations, are established and evolve. ${ }^{13}$ The use of SNA has been suggested for designing and evaluating complex interventions with the goal of understanding and examining complex interactions among or between networks. ${ }^{912} 1^{14-19}$ The aim of this scoping review was to identify and determine the value of SNA in studies that develop or evaluate complex interventions in healthcare research.

The research questions were as follows:

1. In which complex healthcare intervention research phases and level of complexity is SNA used?

2. What value do researchers report in the use of SNA for developing and evaluating complex healthcare interventions?

\section{MATERIALS AND METHODS}

A scoping review was conducted to report a wide search for evidence addressing our research questions without specific quality assessment which is common for scoping reviews. ${ }^{2021}$ After identifying the research question, the following steps were conducted: identifying relevant studies; selecting studies based on predefined inclusion criteria; charting the data; and collating, summarising and reporting the results. Although presented as a series of stages, the process was iterative. Steps were repeated when needed to ensure that the literature was reviewed in a comprehensive way. ${ }^{20}$ The Preferred Reporting Items for Systematic Reviews and Meta-Analyses (PRISMA)

\section{Box 1 Search strategy}

\section{PubMed}

("intervention"[All Fields] OR program[All Fields] OR programme[All Fields] OR ("clinical trials as topic"[MeSH Terms] OR ("clinical"[All Fields] AND "trials"[All Fields] AND "topic"[All Fields]) OR "clinical trials as topic"[All Fields] OR "trial"[All Fields]) OR ("Evaluation"[Journal] OR "Evaluation (Lond)"[Journal] OR "evaluation"[All Fields])) AND ("social network analysis"[All Fields] OR "network analysis"[All Fields]) AND (“2004/01/01”[PDAT] : “2019/04/30”[PDAT])

\section{PsychINF0}

("intervention" OR program OR programme OR trial OR evaluation) AND ("social network analysis" OR "network analysis")

\section{Embase}

('intervention'/exp OR 'intervention' OR 'program'/exp OR program OR programme $\mathrm{OR}$ 'trial'/exp $\mathrm{OR}$ trial $\mathrm{OR}$ 'evaluation'/exp $\mathrm{OR}$ evaluation) AND ('social network analysis'/exp OR 'social network analysis' OR 'network analysis'/exp OR 'network analysis') AND (2004:py OR 2005:py OR 2006:py OR 2007:py OR 2008:py OR 2009:py OR 2010:py OR 2011:py OR 2012:py OR 2013:py OR 2014:py OR 2015:py OR 2016:py OR 2017:py OR 2018:py OR 2019:py) AND ('article'/it OR 'article in press'/it OR 'review'/it) AND [embase]/lim NOT ([embase]/lim AND [medline]/lim)

\section{CINAHL}

("intervention" OR program OR programme OR trial OR evaluation) AND ("social network analysis" OR "network analysis")

Extension for Scoping Reviews 2018 was used to guide the reporting. ${ }^{22}$ Ethical approval or patient consent was not required.

\section{Search strategies}

Intervention-based studies using SNA in the field of healthcare were identified through a systematic search using logical operator-based combinations of key terms to identify potentially relevant publications from the Embase, PsycINFO, CINAHL and PubMed databases. The search strategy included the use of a combination of key terms related to complex health interventions and keywords related to SNA (see box 1). For each database, we worked with a librarian from the healthcare discipline to develop a list of relevant keywords. The database searches were conducted from the third week of April 2019 to the end of April 2019. Reference lists of relevant reviews were hand searched.

\section{Inclusion criteria}

Complex healthcare intervention studies were defined as the earlier described consolidated definition for complex interventions by Guise et at'. Only empirical studies were included when the healthcare intervention was targeted the individual or community level. Interventions targeting institutional networks (which may include federal agencies (eg, CDC), local government agencies (eg, city health departments), non-government organisations and private health organisations (eg, hospitals and healthcare providers) public and population healthcare programmes) were therefore excluded. ${ }^{23}$ Additionally, 


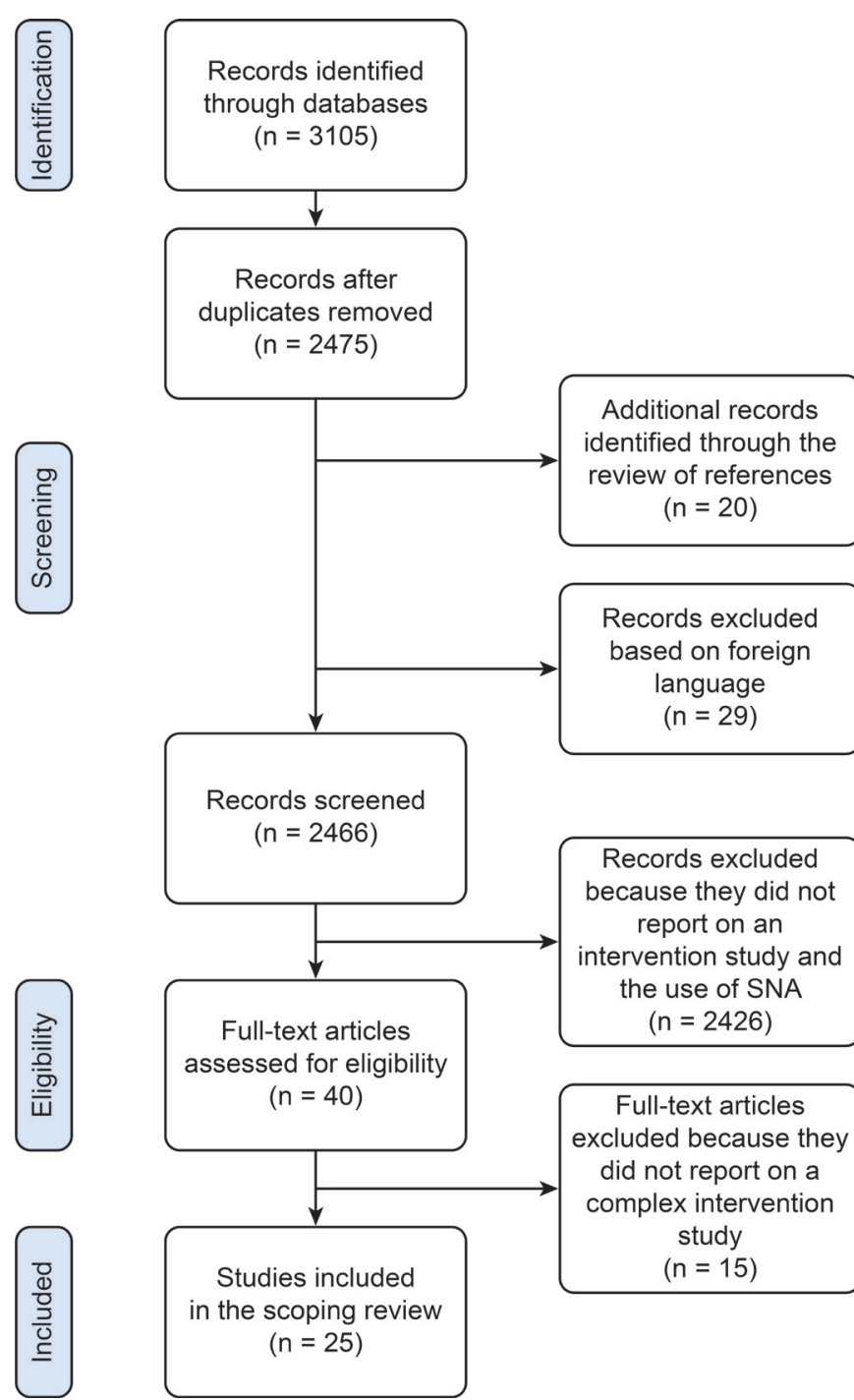

Figure 1 Preferred Reporting Items for Systematic Reviews and Meta-Analyses flow diagram.

studies had to report use of SNA in the design of the study, for example, social network mapping, assessment of network structure and properties, or analysis of network members. Studies were excluded if (1) social networks were mentioned, but the type of analysis was not reported; (2) the primary focus was social support, peer support, social capital or other related topics, but did not report an SNA. Studies published in any language other than English were excluded from the review. The search was limited to studies published between January 2004 and April 2019. This time period was carefully chosen with the goal of including relevant studies from the moment that the use of SNA in research was emerging. ${ }^{12}$ If studies reported the same data in two or more journals, the second and subsequent submissions were excluded. While we did not include (systematic) reviews, we did check the references from these reviews to identify relevant and eligible articles to ensure that we were comprehensive in our search (figure 1). Furthermore, we did not use the study quality as an inclusion criterion. ${ }^{20}$ All studies that met the inclusion criteria were uploaded into
Rayyan $^{\mathrm{R}}$, a web application for systematic reviews that aims to offer researchers a one-stop dashboard to work through the details of their processes while also allowing their collaborators the ability to see each other's work. ${ }^{2425}$

\section{Study selection}

The study selection involved two steps. First, the list of study titles resulting from the various searches was reviewed by two reviewers (LS and JD) independently, and each reference was assigned a value of 'include', 'exclude' or 'maybe'. Second, the reviewers independently assessed the abstracts of the included titles for relevance. In both steps, disagreement between the two reviewers was resolved by consensus, with input from a third author (NB) when necessary.

\section{Data extraction}

Data were extracted from the included studies using a structured format that enabled us to (1) describe the study characteristics, (2) describe the level of complexity of the healthcare interventions (3) report the strengths and limitations of the application of SNA, and (4) report the implications of using SNA in complex interventionbased studies. To describe the study characteristics, data regarding the author, date of publication, country of the study, type of intervention, target of the SNA in the intervention design, SNA purpose and the metrics used were extracted. To describe the level of complexity of the healthcare interventions, data were extracted based on the Complexity Assessment Tool for Systematic Reviews (iCAT_SR). Six core dimensions and two optional dimensions were assessed by defined criteria (see online supplemental appendix 1). ${ }^{26}$ The eight dimensions covered the earlier described consolidated definition for complex interventions in which intervention complexity, implementation complexity, population complexity, pathway complexity and contextual complexity stood central. To describe the value of using SNA for developing and evaluating complex interventions, the strengths and limitations of the application of SNA were extracted from the included studies first. Next, the reported implications of using SNA were extracted. The data extraction process and format were initially piloted by the first two authors with five studies. In the next stage, each author independently extracted data from the remaining studies. After extraction, the data were compared, and differences were discussed between the two reviewers, with input from a third author (NB) when necessary, until agreement was reached.

\section{Collating, summarising and reporting the results}

Following data extraction, a narrative synthesis was created to describe the included studies in terms of the study characteristics, level of complexity of the healthcare interventions, the reported strengths and limitations of the application of SNA, and the reported implications of using SNA in the development and evaluation of complex 
interventions. This narrative was intended to provide an overall description of the available evidence. ${ }^{20}$

\section{Patient and public involvement}

No patients or public were involved in this study.

\section{RESULTS}

\section{Studies identified}

After removing the duplicates, we identified 2466 potentially relevant studies, 20 of which we identified by hand searching. After abstract screening, 40 full-text studies were assessed for eligibility, resulting in 25 studies being included in the review (see figure 1). The publication year of the included complex intervention studies ranged from 2009 to 2019. The countries of origin were diverse; however, 11 studies $(44 \%)$ were conducted in the USA. As shown in table 1, the application of SNA in developing and evaluating complex interventions differed. Most studies $(60 \%)$ used SNA to evaluate (partially) the effectiveness of an intervention. No study used SNA when developing an intervention. In two studies, SNA findings were used to provide information on the feasibility of the complex intervention. ${ }^{27} 28$ The types of interventions, as well as the SNA purpose, were diverse. Most studies identified relationships between actors, while other studies collected data on the specific network type, such as knowledge exchange or patterns of collaboration (table 1) (see online supplemental appendix 2 for the extended study characteristics and online supplemental appendix 3 for the application of SNA in the included studies).

\section{Level of complexity of included studies}

The level of complexity of the included studies based on the iCAT_SR is shown in table $2 .{ }^{27}$ Regarding the intervention complexity, only two studies reported one component intervention ${ }^{29} 30$ while the other studies consisted of a multicomponent intervention whether or not offered as a bundle. Behaviour or actions of intervention recipients of the studies were divers from single till dual or multiple target. The implementation complexity showed that the degree of tailoring the intervention was in 10 studies inflexible (40\%), 11 studies moderate $(44 \%)$ and in 4 studies highly flexible $(16 \%)$. The level of skill required by those delivering the intervention was in most studies intermediate $(84 \%)$ and for those receiving the intervention, was the level of skills required basic in most studies $(88 \%)$. The population complexity was low in 16 studies $(64 \%)$ as the interventions directed only at single category of individuals within the individual level (eg, professionals or patients), 5 studies (20\%) were defined as multicategory as the interventions directed at 2 or more categories of individuals within the individual level (eg, primary care professionals and primary care patients), 4 studies $(16 \%)$ were defined as multilevel as the intervention directed at 2 or more levels. The pathway complexity was in $21(84 \%)$ studies unclear or unable to assess, only $4(16 \%)$ studies used a logic model to explain the nature of the causal pathway between the intervention and the outcome it is intended to effect. Three studies (12\%) were defined as having a long variable pathway and one study (4\%) having a short, linear path. Contextual complexity was, except for two studies (4\%) which interventions could moderately dependent on individual-level factors, unclear or unable to assess.

\section{Reported strengths and limitations of the application of SNA}

Table 3 provides an overview of the reported strengths and limitations. Of the included studies, 6 studies (24\%) reported only strengths in the application of SNA for developing and evaluating complex interventions, ${ }^{31-36} 1$ study $(4 \%)$ reported a limitation, ${ }^{37} 4$ studies (16\%) did not report any strength or limitation, ${ }^{38-41}$ and the remaining 14 studies $(56 \%)$ reported strengths as well as limitations in the application of SNA. Reported limitations of the application of SNA were focused on the study design and data collection. Regarding the study design, the lack of a qualitative component and lack of control group were reported as limitations because they prevent more in-depth understanding of the results and contribute to lower methodological rigour than that of some other analysis methods, which inhibits authors from stating the causal effects of an intervention. ${ }^{27} 283642$ A mixedmethod approach was reported as a strength for gaining an in-depth understanding of the results. ${ }^{31}$ Reported limitations related to data collection were possible recall bias due to self-reported data, the challenge of obtaining responses, and non-respondent data. ${ }^{19}$ 28-30 $3743-46$ The absence of nonrespondent data may introduce potential bias and can therefore dramatically affect network representation. ${ }^{19}$ Reported strengths were that SNA data are easy to collect ${ }^{28}$ and that data can be collected by various methods, ${ }^{19}$ including specific SNA tools (NET map, Social Network Diagnostic Tool and Partner Tool). ${ }^{31} 4047$ Regarding analysis, the quantitative results that SNA yields can be combined with other statistical approaches. ${ }^{34}$ In addition, sociometrics may have superior value for overcoming the shortcomings of ego network self-reported measures, but data collection from ego networks is more feasible and less expensive than sociometric network data collection. ${ }^{44}$ SNA analysis is further strengthened because it is based on the number of relationships instead of only the number of individuals. ${ }^{43}$ Additionally, the use of SNA programmes to analyse data was reported as a strength in terms of the ease of use but as a limitation in terms of the need for special training and experience. ${ }^{19} 2744$ The visualisation of SNA results can strengthen the interpretation of the results. ${ }^{44}$ However, a reported limitation was that complexity cannot be captured in simplified visuals. ${ }^{19}$ Additionally, the interpretation of the results was reported as strength, as SNA provides insights into the relationships, positions, structure and strength of a network. ${ }^{19} 3148$ 
Table 1 Study characteristics

Target of SNA

Intervention

type

SNA purpose

Pilot/feasibility phase

Study Identification of Acceptability Interventionists
Implementation

phase

Evaluation phase

Implementation

evaluation

Banbury et $a l^{37}$

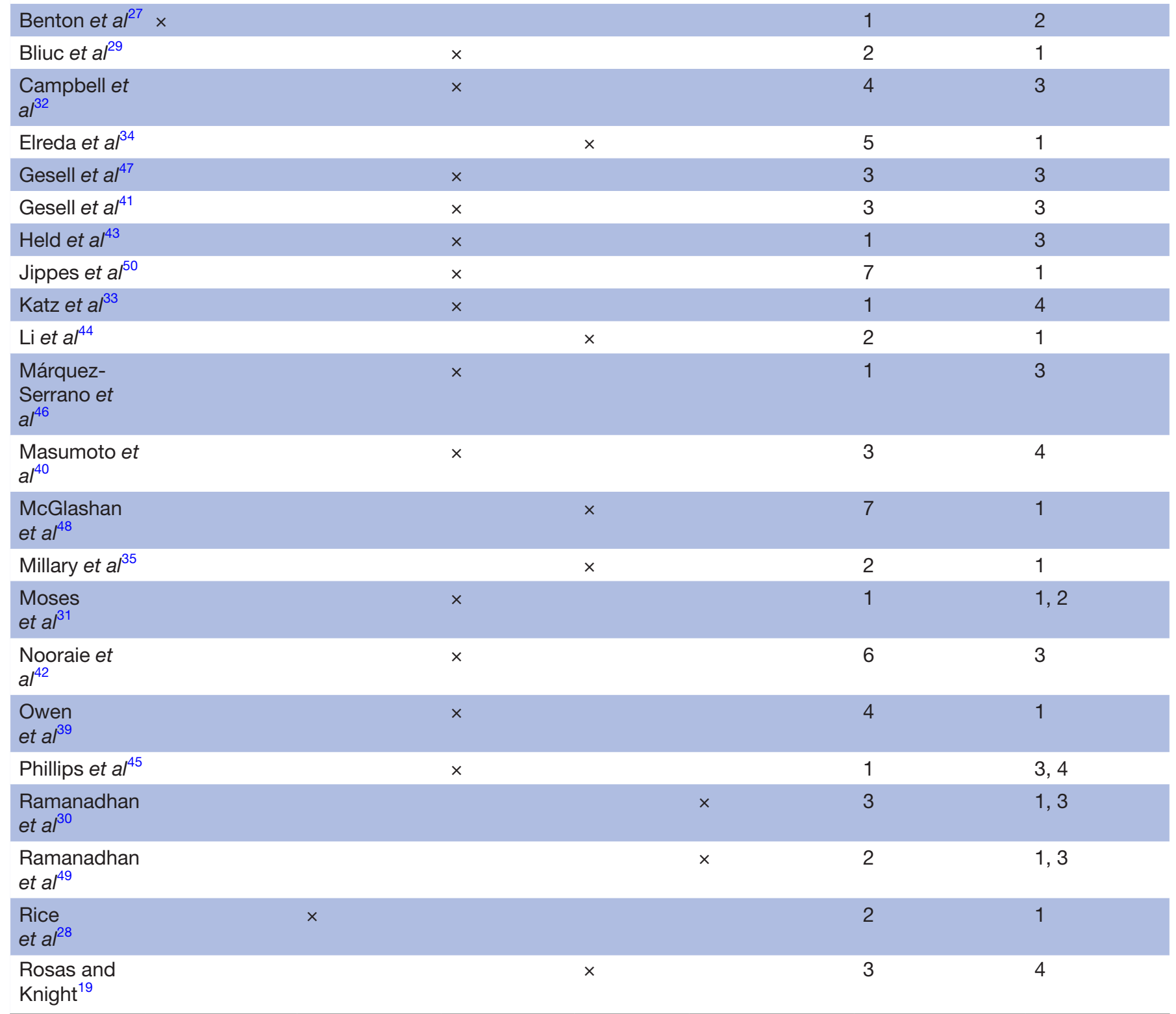


Target of SNA

\begin{tabular}{|c|c|c|c|c|c|}
\hline & & & & & \\
\hline & Pilot/feasibility phase & Evaluation phase & $\begin{array}{l}\text { Implementation } \\
\text { phase }\end{array}$ & & \\
\hline $\begin{array}{l}\text { Spitzer- } \\
\text { Shohat et }\left.a\right|^{36}\end{array}$ & & & $\times$ & 8 & 1 \\
\hline Yang et $a l^{38}$ & & $\times$ & & 9 & 1 \\
\hline
\end{tabular}

However, the generalisability of SNA results is limited due to the unique nature of a network. ${ }^{48} 49$

\section{Reported implications and added value of SNA}

Fifteen studies reported implications of using SNA in developing and evaluating complex interventions. $192728303133353643-4648-51$ Three studies reported the wider use of SNA in their topic of research, namely, interprofessional education, train-the-trainer programmes and the evaluation of nursing interventions. ${ }^{27} 4546$ Figure 2 shows a graphical framework that summarises reported strengths of the application of SNA and reported implications, and connects their content to the dimensions of complexity. The graphical framework depicts the ways SNA can be used in the various phases of complex intervention research in healthcare, in connection to complexity of the intervention, implementation, population, pathway and context.

Regarding the development phase, the acceptability study by Rice $e t a l^{28}$ reported that SNA can provide essential information in the design of large-scale efficacy studies. For the pilot phase, the educational intervention by Benton et $a l^{27}$ indicated that SNA offers an opportunity to introduce quantitative rigour to the selection of interventionists. Rice $e t a t^{28}$ suggested that the identified people can disseminate innovations. SNA results can also inform the design of feasibility trials. ${ }^{50}$ In regard to the evaluation phase, five studies reported implications. 3033354344 One study that was characterised as an implementation study reported that SNA provides useful monitoring and evaluation data for both evaluation and implementation purposes. ${ }^{30}$ The process evaluation study by Millery et $a l^{5}$ suggested that SNA allows analysis of the network as a whole system and at the individual organisation level. Such analysis enables researchers to document systemic change beyond simple shifts in knowledge, attitudes and skills. Both levels were reported to be very useful for an evaluation framework in a transactional context. ${ }^{35}$ Some authors reported that SNA can measure network structural factors beyond the intervention, which is necessary to understand the broader context. ${ }^{44}$ Furthermore, the effectiveness study by Held et $a l^{43}$ reported that SNA helps to identify points of leverage to create and improve targeted intervention strategies. For the implementation phase, the reported implications indicated that SNA provides an in-depth understanding of the barriers and/ or facilitators of the diffusion and implementation of an intervention. SNA also offers actionable insights into the network of interest, such as insights into skill transfer and team effectiveness, which can guide the implementation of large-scale efficacy studies. ${ }^{28} 36$

\section{DISCUSSION}

This scoping review described the specific use of SNA in different phases of complex intervention research, in different level of intervention complexity, as well as the value of using SNA for developing and evaluating complex interventions. Five main conclusions can be drawn from this analysis. First, SNA seems underused in evaluating complex intervention research. Second, SNA was not used in the development phase of the included studies. Third, the reported implications in the evaluation and implementation phase reflect the value of SNA in addressing the implementation and population complexity. Fourth, pathway complexity and contextual complexity of the included interventions were unclear or unable to access. Fifth, the use of a mixed methods approach was reported as a strength, as the combination and integration of a quantitative and qualitative method clearly establish the results.

This study showed that SNA seems underused in evaluating complex intervention research. A total of 25 complex healthcare intervention-based studies published in the last 10 years in the field of healthcare were found that used SNA. This number is comparable to the findings of a systematic review reporting the application of SNA in health behaviour intervention studies. ${ }^{51}$ SNA has developed only over the past 20 years from a niche discipline in sociology to an approach applied in many fields of the physical and biological sciences. ${ }^{52}$ SNA is focused on the structure of relationships and assumes that relationships are important. ${ }^{53}$ Most complex interventions are embedded within a network of multiple (healthcare) professionals from multiple sectors and disciplines. ${ }^{11}$ Recent studies, therefore, highlight the importance of understanding and examining networks and their interactions in complex intervention research. ${ }^{9} 12$ 14-19

Although SNA has been used in the pilot, evaluation and implementation phase, this study showed that no study used SNA in the development phase. Several frameworks are available for the development and evaluation of complex interventions. ${ }^{154-58}$ Optimising the development 


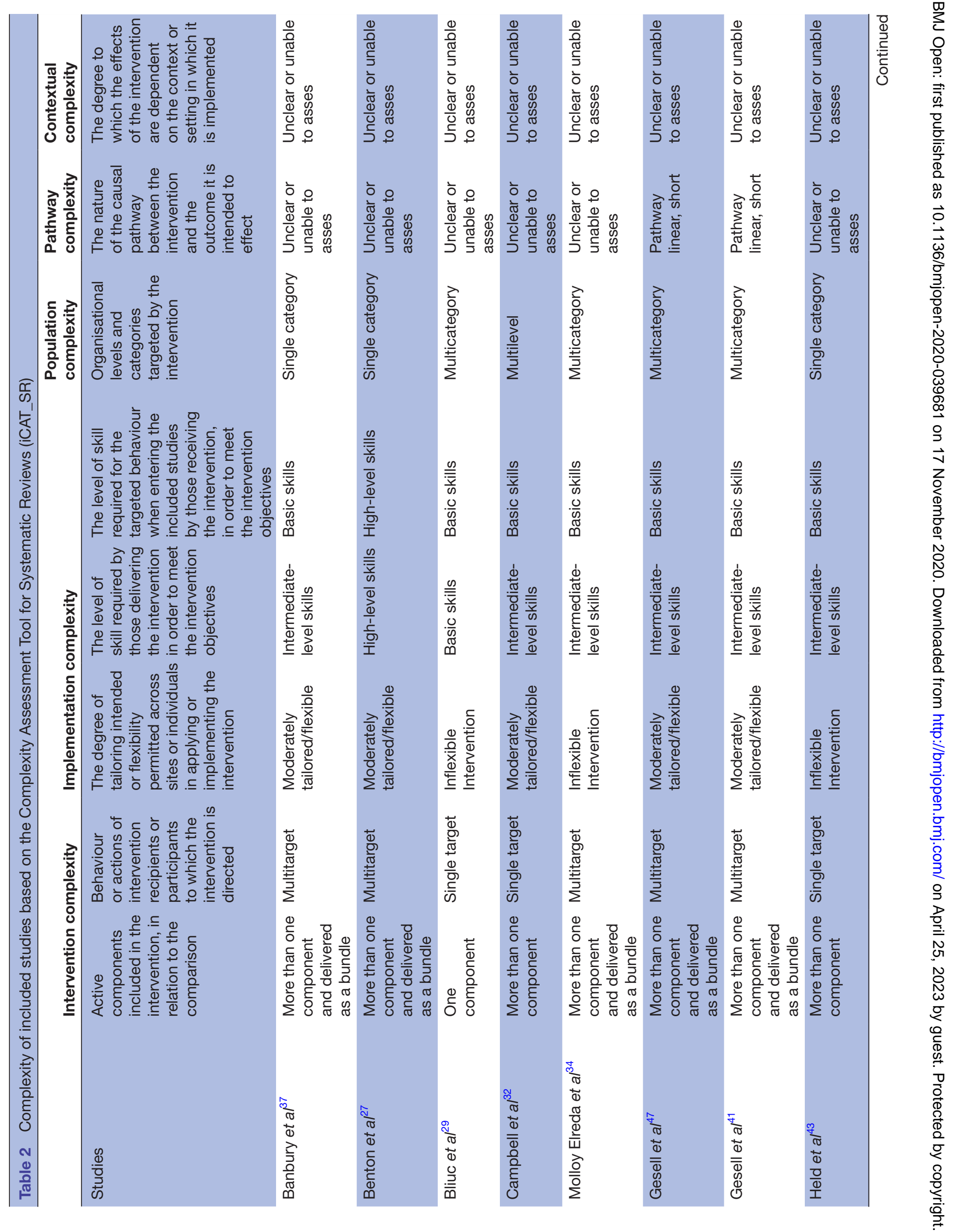




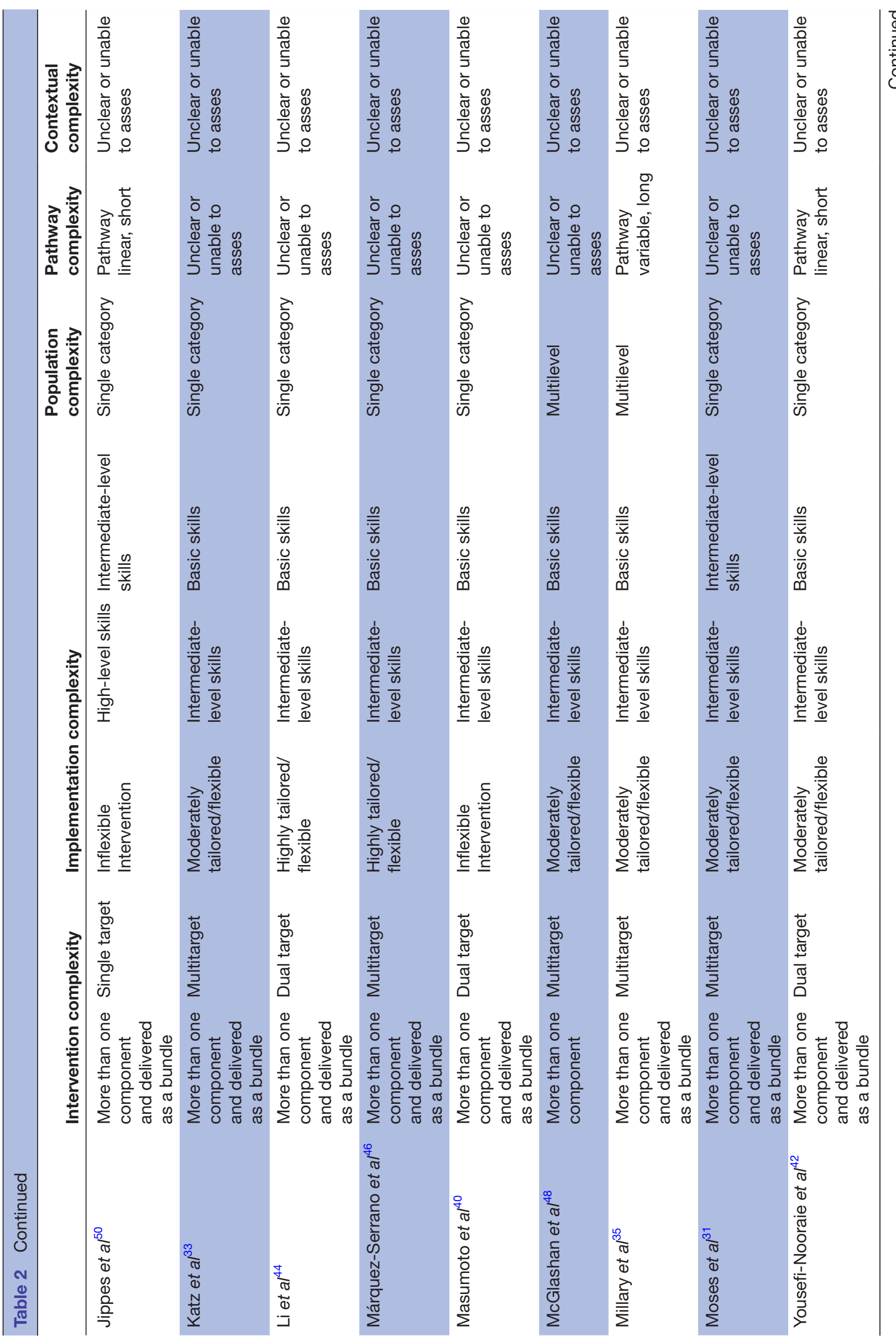




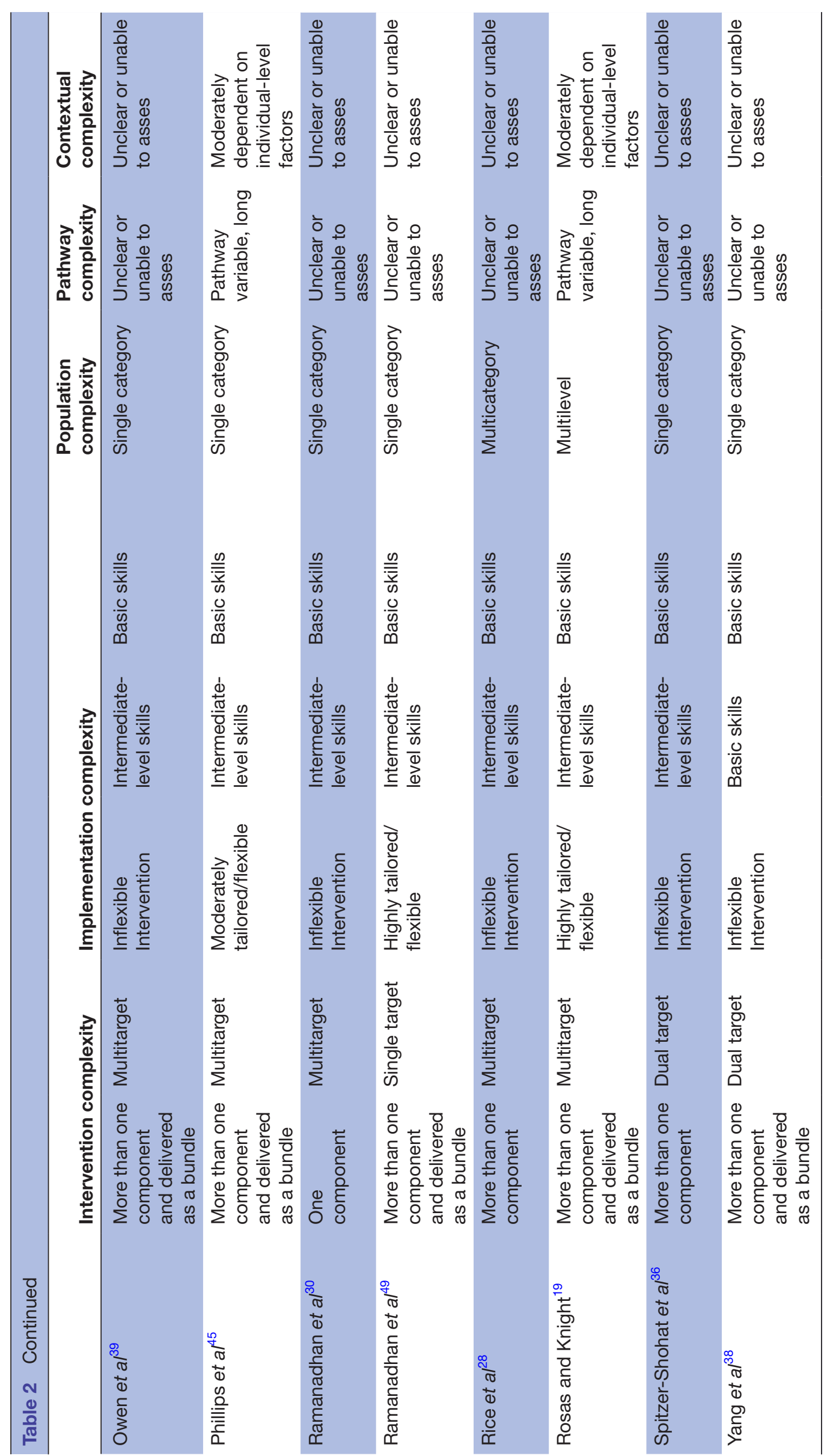


Table 3 Reported strengths and limitations in the application of social network analysis (SNA) in complex intervention research

\begin{tabular}{ll}
\hline Application component & Strengths \\
\hline Design & \\
SNA as a method & \\
& SNA 31424449 \\
& traditional analysis and generates new \\
& information. \\
& SNA reveals important intervention \\
& dynamics that would not be found with \\
& classical methods. \\
& SNA moves beyond individual-level effects \\
& and captures system-level effects. \\
& Longitudinal SNA can reveal underlying \\
& social processes after the implementation of \\
& the intervention.
\end{tabular}

Type of SNA method 272936 A mixed methods approach clearly establishes the results.

Type of SNA method 27 36

\section{Limitations}

Control group 2842

\begin{tabular}{|c|c|}
\hline Data & \\
\hline Data collection ${ }^{19} 28-303743-46$ & $\begin{array}{l}\text { Data are easily to collect. } \\
\text { Primary data can be collected through } \\
\text { several methods such as surveys, } \\
\text { workshops or interviews. }\end{array}$ \\
\hline
\end{tabular}

\section{SNA tools (NET map, Socia Network Diagnostic Tool, Partner Tool) ${ }^{32} 3547$}

NET map is a tool for action research that yields visual quantitative and qualitative evaluation data; it enhances the sense of a shared purpose among network members.

- A social network diagnostic tool can monitor group programmes during implementation and can guide programme activities with the intent to build new social networks.

- The SNA Partner Tool produces a rich set of network metrics to describe the state of the network at baseline.
- The lack of a qualitative component results in a less comprehensive understanding of the results.

- Insight into the structure does not indicate causality.

- Due to the lack of a parallel control group, findings on the changes in social networks through the implementation of the intervention could simply be the result of natural tendencies in social networks over time and not the effect of the intervention per se.

- The data collection method can be restrictive in examining relations involving more than two people.

- Self-reported data induce recall bias.

- There is a possibility for social desirability bias.

- Obtaining responses for (longitudinal) data collection can be challenging.

- The operationalisation of the network type of interest can be interpreted in multiple ways.

- Constructing sociometric network data requires outreach work and knowledge of the community.

- Egocentric network data collection is much more feasible and less expensive than sociometric network data collection.

- The Social Network Diagnostic Tool is not sensitive to the measurement of different mechanisms explaining social influences. 
Table 3 Continued

\begin{tabular}{|c|c|c|}
\hline Application component & Strengths & Limitations \\
\hline Non-respondent data ${ }^{192848}$ & & $\begin{array}{l}\text { The absence of non-respondent data } \\
\text { may introduce potential bias, as non- } \\
\text { respondents' positions in the network may } \\
\text { lead to them being difficult to contact in } \\
\text { retrospect. Alternatively, the occurrence } \\
\text { of missing data may be random due to } \\
\text { staff turnover and changing contact details } \\
\text { between the end of the intervention and the } \\
\text { data collection period. } \\
\text { Missing or erroneous data can dramatically } \\
\text { affect network representation. }\end{array}$ \\
\hline
\end{tabular}

$\begin{array}{ll}\text { Analysis } & \text { SNA provides a wide range of tools for } \\ \text { Quantitative metrics } & 2734 \\ & \text { quantifying the structure and strengths of } \\ & \text { networks (of interest) during an intervention. } \\ & \text { SNA can support multiple analyses of } \\ & \text { effectiveness at the individual level. } \\ & \text { SNA can be combined with other statistical } \\ & \text { approaches. }\end{array}$

\begin{tabular}{|c|c|}
\hline Sociometrics ${ }^{3044}$ & $\begin{array}{l}\text { Sociometrics have superior value in } \\
\text { overcoming the shortcomings of ego } \\
\text { network self-reported measures. } \\
\text { Sociometrics strengthen studies. }\end{array}$ \\
\hline
\end{tabular}

Use of SNA programmes (eg, The use of programmes as UCINET and UCINET, NETDRAW) ${ }^{19} 2744$

\section{Number of respondents ${ }^{43}$ SNA is focused on relationships instead of individuals (the number of respondents), which establishes the basis for the quantitative analysis (power).}

Results

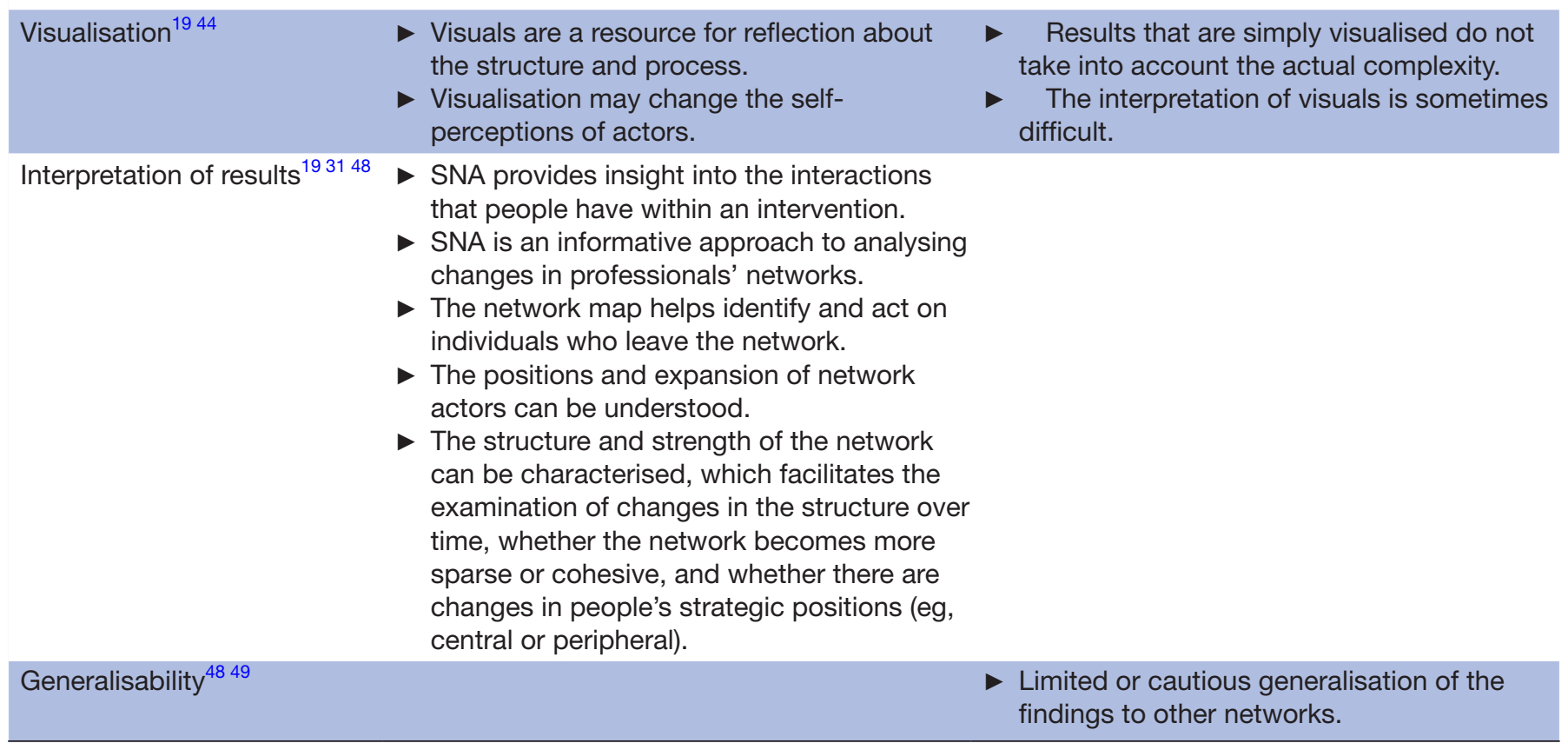

Network data analysis requires special training.

SNA requires experience. 


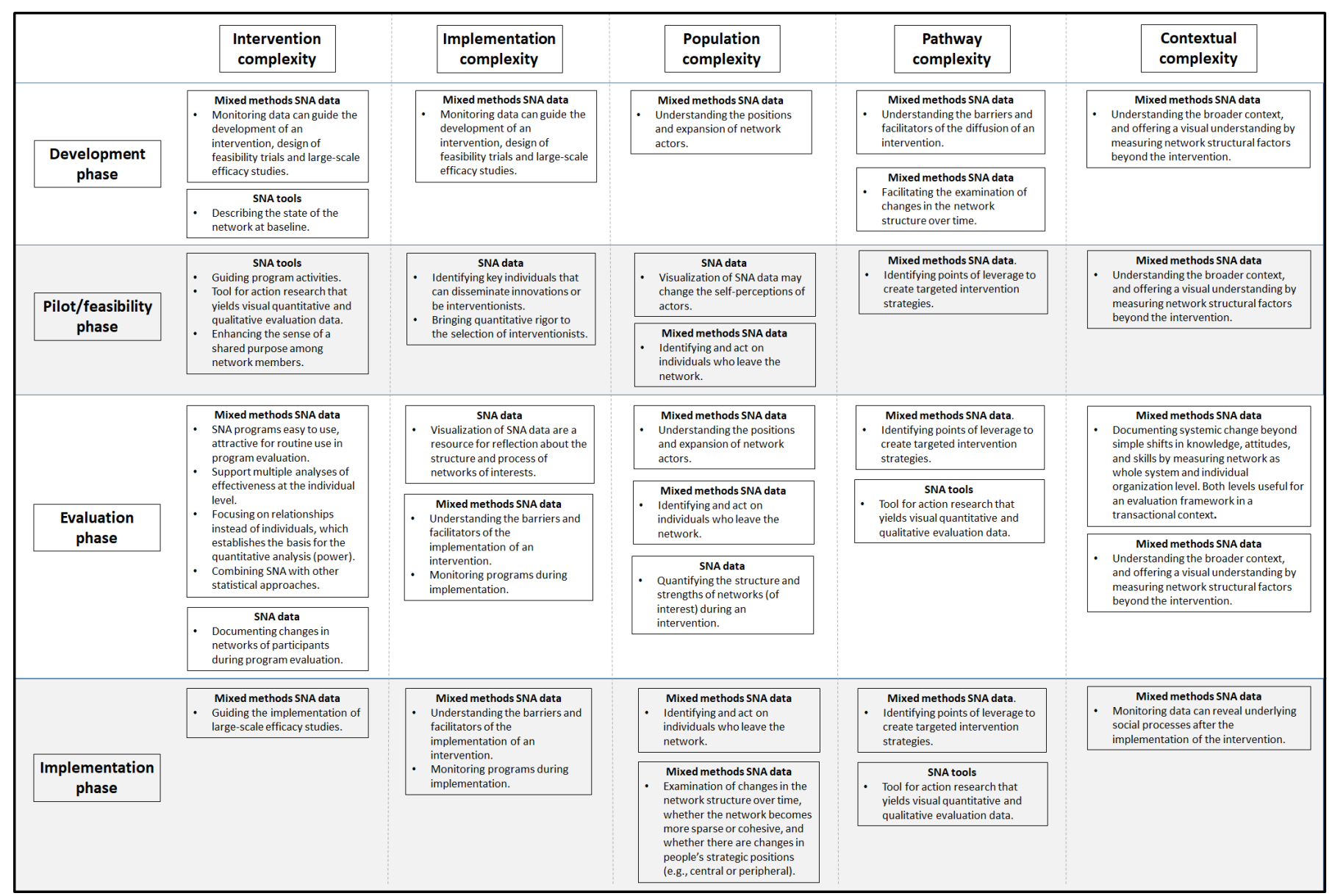

Figure 2 Graphical framework. SNA, social network analysis.

of a complex intervention will enhance the intervention design, increase value and minimise the risk of subjects being exposed to ineffective interventions. A gap between the intervention and the implementation context often results in suboptimal treatment success ${ }^{59}$ and SNA may contribute to bridge the gap and to understand the implementation context. During the development phase, SNA can provide strategies to consider the social context of programme delivery, determine the appropriate methods and communication needs, and identify particular change agents and opinion leaders in the network to focus on ${ }^{60-62}$ This suggests that SNA can be of great value when developing, complex interventions.

This study shows a significant potential of using SNA in addressing the implementation and population complexity in various ways. Although frameworks highlight the importance of a systematic development and evaluation of complex interventions, an iterative rather than linear process is recommended. ${ }^{154-58}$ An iterative process allows researchers to consider the implementation complexity and population complexity prior to the implementation. When addressing implementation complexity, SNA could focus (1) on the skills required by the intervention providers who deliver the intervention and (2) the tailoring carried out by the intervention providers, regarding the receiver or context, in applying or implementing the intervention. ${ }^{26}$ By addressing the population complexity, SNA can highlight the structures of the organisational levels and categories targeted by the intervention. ${ }^{26}$ Since interventions itself might alter networks and since networks are dynamic and likely to change over time, researchers are therefore encouraged to collect network and outcome data of interest longitudinally (eg, monitoring data) and cross-sectionally. ${ }^{43} 51$ Furthermore, the use of monitoring SNA data can identify points of leverage to create and improve targeted intervention strategies. ${ }^{43}$ Valente $e t a b^{60}$ published a practical overview of how to use SNA for programme implementation to understand which social network can be created, maintained and accomplished.

This study showed that the pathway complexity and contextual complexity of most interventions were unclear or unable to access. The limited reporting regarding the use of a logic model which describe the nature of the causal pathway between the intervention and its effect, and contextual factors which can influence the effectiveness of an intervention, was also observed by Smit $e t a l^{10}$ that examined complex primary healthcare interventions. ${ }^{10}$ SNA could be of value in addressing the contextual complexity as the degree to which the effects of the intervention are dependent on the context or setting in which it is implemented. ${ }^{26}$ The Consolidated Framework 
for Implementation Research (CFIR) comprises 39 constructs organised across five major domains (eg, intervention, outer setting, inner setting, characteristics of individuals and process), all of which interact to influence implementation and implementation effectiveness. ${ }^{5}$ The study of Kirk $e t a t^{6}$ provide a broad overview of CFIR constructs used in literature which can be directory in disentangling and addressing contextual complexity. In general, the graphic framework, introduced in this study, is a first step and can be used in future research in this area. Additionally, more research is needed to assess the optimal way to use SNA in complex intervention research in healthcare, especially in relation to the five dimensions of complexity.

The included studies reported the use of a mixed methods approach to be a strength, as the combination and integration of a quantitative and qualitative method clearly establishes the results. All included studies were quantitative studies, consistent with their use of SNA, which is quantitative in nature. A strong reliance on quantitative methods was criticised. Adding a qualitative approach alongside quantitative procedures can be a solution to generate an in-depth understanding of the results. ${ }^{63}{ }^{64}$ SNA increasingly relies on both quantitative and qualitative approaches for data collection and analysis. ${ }^{65}$ The development and evaluation of complex interventions often require multiple research questions which reflect the number of behaviours or actions that the intervention focuses on as part of the intervention complexity. ${ }^{26}$ The use of mixed methods social network analysis (MMSNA) can be an appropriate means to answer these research questions in which the '13-step model' of Schooneboom (2018) can guide researchers. ${ }^{66}$ Although the use of MMSNA is recommended, MMSNA still lacks conceptual clarity as, as the 'when', 'how' and 'why' of a mixed methods approach are rarely described. ${ }^{67}$ However, MMSNA seems promising, and a mixed-method approach is consistent with the multiphase model of complex intervention development and evaluation.

\section{Strengths and limitations}

This study has several strengths. First, the data collection and data management processes were thorough. Two researchers selected the studies in accordance with the inclusion and exclusion criteria, which were determined beforehand. In addition, the data charting and synthesis of the results were also conducted by two researchers (LS and JD) working independently. The synthesis of the results was checked and confirmed by all authors to ensure the validity of the findings. Second, the literature search was conducted in four scientific databases, which is more than sufficient to include the central and relevant research evidence in healthcare ${ }^{68}$ In addition, the reference lists of the reviews in our search were hand searched to identify studies that otherwise potentially would have been missed. Third, the review process followed a universally agreed protocol (PRISMA Extension for Scoping Reviews 2018) to ensure the quality of reporting. ${ }^{22}$
Additionally, in the analysis, the authors' original expressions were used without any interpretations.

This review has some limitations. First, there is no sharp boundary between simple and complex healthcare interventions. ${ }^{1}$ To overcome this limitation, the level of complexity was unravelled of all included studies based on the Icat_SR. ${ }^{26}$ Second, this study did not critically appraise the included studies. However, the literature states that scoping reviews cannot identify gaps in the literature related to the low quality of research. ${ }^{69} 70$ By not addressing the issues of quality appraisal, this study was able to include a larger range of study designs and methodologies than would have been included in a systematic review ${ }^{71}$; thus, the emphasis of a scoping study is on comprehensive coverage rather than a particular standard of evidence. ${ }^{72}$

\section{CONCLUSION}

Based on the application of SNA in 25 studies, we conclude that SNA is a valuable method to apply, but currently underused. SNA has been applied in the pilot, evaluation and implementation phases of complex intervention research. Although there is an absence of studies applying SNA in the development of complex interventions, the included studies reported the potential value of SNA in the development phase. Furthermore, SNA can be of value to disentangle and address the five dimensions of complexity of complex healthcare interventions. The routine use of SNA within a mixed-method approach for developing and evaluating complex interventions could yield actionable insights that would be useful in the transactional context of complex interventions.

\section{Author affiliations}

${ }^{1}$ Research Centre for Healthy and Sustainable Living, University of Applied Sciences Utrecht, Utrecht, The Netherlands

${ }^{2}$ Faculty of Health, Nutrition \& Sport, The Hague University of Applied Sciences, The Hague, The Netherlands

${ }^{3}$ Education Center, UMC Utrecht Academy, University Medical Center Utrecht, Utrecht, The Netherlands

${ }^{4}$ Department of General Practice, Division Julius Center for Health Sciences and Primary Care, University Medical Center Utrecht, Utrecht, The Netherlands

${ }^{5}$ Department of Nursing Science, Julius Centre for Health Sciences and Primary Care, University Medical Center Utrecht, Utrecht, The Netherlands

Contributors LS and JD contributed to this article by conducting the design, data collection, data analysis and by reviewing the manuscript. MJS, NJdW and NB contributed to this article by conducting the design, and by reviewing the manuscript. All authors approved the manuscript and this submission.

Funding The authors have not declared a specific grant for this research from any funding agency in the public, commercial or not-for-profit sectors.

Competing interests None declared.

Patient consent for publication Not required.

Provenance and peer review Not commissioned; externally peer reviewed.

Data availability statement All data relevant to the study are included in the article or uploaded as supplementary information.

Supplemental material This content has been supplied by the author(s). It has not been vetted by BMJ Publishing Group Limited (BMJ) and may not have been peer-reviewed. Any opinions or recommendations discussed are solely those of the author(s) and are not endorsed by BMJ. BMJ disclaims all liability and 
responsibility arising from any reliance placed on the content. Where the content includes any translated material, BMJ does not warrant the accuracy and reliability of the translations (including but not limited to local regulations, clinical guidelines, terminology, drug names and drug dosages), and is not responsible for any error and/or omissions arising from translation and adaptation or otherwise.

Open access This is an open access article distributed in accordance with the Creative Commons Attribution Non Commercial (CC BY-NC 4.0) license, which permits others to distribute, remix, adapt, build upon this work non-commercially, and license their derivative works on different terms, provided the original work is properly cited, appropriate credit is given, any changes made indicated, and the use is non-commercial. See: http://creativecommons.org/licenses/by-nc/4.0/.

\section{ORCID iD}

Linda C Smit http://orcid.org/0000-0001-5488-2967

\section{REFERENCES}

1 Craig P, Dieppe P, Macintyre S, et al. Developing and evaluating complex interventions: the new medical Research Council guidance. BMJ 2008;337:a1655.

2 Guise J-M, Chang C, Viswanathan M, et al. Agency for healthcare research and quality evidence-based practice center methods for systematically reviewing complex multicomponent health care interventions. J Clin Epidemiol 2014;67:1181-91.

3 Petticrew M, Anderson L, Elder R, et al. Complex interventions and their implications for systematic reviews: a pragmatic approach. $J$ Clin Epidemiol 2013;66:1209-14.

4 Guise J-M, Butler M, Chang C, et al. AHRQ series on complex intervention systematic reviews-paper 7: PRISMA-Cl elaboration and explanation. J Clin Epidemiol 2017;90:51-8.

5 Damschroder LJ, Aron DC, Keith RE, et al. Fostering implementation of health services research findings into practice: a consolidated framework for advancing implementation science. Implement Sci 2009;4:50.

6 Kirk MA, Kelley C, Yankey N, et al. A systematic review of the use of the consolidated framework for implementation research. Implement Sci 2015;11:72.

7 Wells M, Williams B, Treweek S, et al. Intervention description is not enough: evidence from an in-depth multiple case study on the untold role and impact of context in randomised controlled trials of seven complex interventions. Trials 2012;13:95

8 Craig P, Ruggiero ED, Frohlich KL, et al. Taking account of context in population health intervention research: guidance for producers, users and funders of research. Canada: Canadian Institutes of Health Research, 2018.

9 Moore GF, Evans RE, Hawkins J, et al. From complex social interventions to interventions in complex social systems: future directions and unresolved questions for intervention development and evaluation. Evaluation 2019;25:23-45.

10 Smit LC, Schuurmans MJ, Blom JW, et al. Unravelling complex primary-care programs to maintain independent living in older people: a systematic overview. J Clin Epidemiol 2018;96:110-9.

11 Leischow SJ, Milstein B. Systems thinking and modeling for public health practice. Am J Public Health 2006;96:403-5.

12 Benton DC, Pérez-Raya F, Fernández-Fernández MP, et al. A systematic review of nurse-related social network analysis studies. Int Nurs Rev 2015;62:321-39.

13 Scott J. Social network analysis. Thousand Oaks, CA: Sage, 2017.

14 Pow J, Gayen K, Elliott L, et al. Understanding complex interactions using social network analysis. J Clin Nurs 2012;21:2772-9.

15 Parnell JM, Robinson JC. Social network analysis: presenting an underused method for nursing research. J Adv Nurs 2018;74:1310-8.

16 Hawe P. Lessons from complex interventions to improve health. Annu Rev Public Health 2015;36:307-23.

17 Keogh F, Pierce M, Neylon K, et al. Intensive home care packages for people with dementia: a realist evaluation protocol. BMC Health Serv Res 2018;18:829.

18 Loutfi D, Andersson N, Law S, et al. Reaching marginalized young women for HIV prevention in Botswana: a pilot social network analysis. Glob Health Promot 2020;27:74-81.

19 Rosas S, Knight E. Evaluating a complex health promotion intervention: case application of three systems methods. Crit Public Health 2019;29:337-52.

20 Arksey H, O'Malley L. Scoping studies: towards a methodological framework. Int J Soc Res Methodol 2005;8:19-32.

21 Levac D, Colquhoun H, O'Brien KK. Scoping studies: advancing the methodology. Implement Sci 2010;5:69.
22 Tricco AC, Lillie E, Zarin W, et al. PRISMA extension for scoping reviews (PRISMA-ScR): checklist and explanation. Ann Intern Med 2018;169:467-73.

23 Bauermeister JA, Tross S, Ehrhardt AA. A review of HIV/AIDS system-level interventions. AIDS Behav 2009;13:430-48.

24 Ouzzani M, Hammady H, Fedorowicz Z, et al. Rayyan-a web and mobile APP for systematic reviews. Syst Rev 2016;5:210.

25 Johnson N, Phillips M. Rayyan for systematic reviews. J Electron Resour Librariansh 2018;30:46-8.

26 Lewin S, Hendry M, Chandler J, et al. Assessing the complexity of interventions within systematic reviews: development, content and use of a new tool (iCAT SR). BMC Med Res Methodol 2017;17:76.

27 Benton DC, Fernández Fernández MP, Fernández MPF. Social network analysis: a tool for the identification of next generation trainers. Collegian 2014;21:311-8.

28 Rice E, Tulbert E, Cederbaum J, et al. Mobilizing homeless youth for HIV prevention: a social network analysis of the acceptability of a face-to-face and online social networking intervention. Health Educ Res 2012;27:226-36.

29 Bliuc A-M, Best D, lqbal M, et al. Building addiction recovery capital through online participation in a recovery community. Soc Sci Med 2017;193:110-7.

30 Ramanadhan S, Wiecha JL, Gortmaker SL, et al. Informal training in staff networks to support dissemination of health promotion programs. Am J Health Promot 2010;25:12-18.

31 Moses AS, Skinner DH, Hicks E, et al. Developing an educator network: the effect of a teaching scholars program in the health professions on networking and productivity. Teach Learn Med 2009;21:175-9.

32 Campbell N, Schiffer E, Buxbaum A, et al. Taking knowledge for health the extra mile: participatory evaluation of a mobile phone intervention for community health workers in Malawi. Glob Health Sci Pract 2014;2:23-34.

33 Katz ML, Pennell ML, Dignan MB, et al. Assessment of cancer education seminars for appalachian populations. J Cancer Educ 2012;27:287-93.

34 Molloy Elreda L, Coatsworth JD, Gest SD, et al. Understanding process in group-based intervention delivery: social network analysis and intra-entity variability methods as windows into the "black box". Prev Sci 2016;17:925-36.

35 Millery M, Aguirre AN, Kukafka R. Does a communityengaged health informatics platform facilitate resource connectivity? an evaluation framework. AMIA Annu Symp Proc 2017;2017:1292-301.

36 Spitzer-Shohat S, Goldfracht M, Key C, et al. Primary care networks and team effectiveness: the case of a large-scale quality improvement disparity reduction program. J Interprof Care 2019;33:472-80.

37 Banbury A, Chamberlain D, Nancarrow S, et al. Can videoconferencing affect older people's engagement and perception of their social support in long-term conditions management: a social network analysis from the telehealth literacy project. Health Soc Care Community 2017;25:938-50.

38 Yang L, Chou Y, Hsu P. Evaluation of on-job training in a ward: nurses job satisfaction and professional commitment. latest advances in educational technologies. in: proceedings of the 11th WSEAS International conference on education and educational technology. Singapore: World Scientific and Engineering Academy and Society Press, 2012: 71-6.

39 Owen JE, Curran M, Bantum Erin O'Carroll, Bantum EO, et al. Characterizing social networks and communication channels in a web-based peer support intervention. Cyberpsychol Behav Soc Netw 2016;19:388-96.

40 Masumoto K, Yaguchi T, Matsuda H, et al. Measurement and visualization of face-to-face interaction among communitydwelling older adults using wearable sensors. Geriatr Gerontol Int 2017; 17:1752-8.

41 Gesell SB, Barkin SL, Sommer EC, et al. Increases in network ties are associated with increased cohesion among intervention participants. Health Educ Behav 2016:43:208-16.

42 Yousefi-Nooraie R, Dobbins M, Marin A, et al. The evolution of social networks through the implementation of evidence-informed decisionmaking interventions: a longitudinal analysis of three public health units in Canada. Implement Sci 2015;10:166.

43 Held FP, Roberts C, Daly M, et al. Learning relationships in community-based service-learning: a social network analysis. BMC Med Educ 2019;19:113.

$44 \mathrm{Li} \mathrm{J}$, Weeks MR, Borgatti SP, et al. A social network approach to demonstrate the diffusion and change process of intervention from peer health advocates to the drug using community. Subst Use Misuse 2012;47:474-90. 
45 Phillips CB, Hall S, Irving M. Impact of interprofessional education about psychological and medical comorbidities on practitioners' knowledge and collaborative practice: mixed method evaluation of a national program. BMC Health Serv Res 2016;16:465.

46 Márquez-Serrano M, González-Juárez X, Castillo-Castillo LE, et al. Social network analysis to evaluate nursing interventions to improve self-care. Public Health Nurs 2012;29:361-9.

47 Gesell SB, Barkin SL, Valente TW. Social network diagnostics: a too for monitoring group interventions. Implement Sci 2013;8:116.

48 McGlashan J, Nichols M, Korn A, et al. Social network analysis of stakeholder networks from two community-based obesity prevention interventions. PLoS One 2018;13:e0196211.

49 Ramanadhan S, Minsky S, Martinez-Dominguez V, et al. Building practitioner networks to support dissemination and implementation of evidence-based programs in community settings. Trans/ Behav Med 2017;7:532-41.

50 Jippes E, Achterkamp MC, Brand PLP, et al. Disseminating educational innovations in health care practice: training versus socia networks. Soc Sci Med 2010;70:1509-17.

51 Shelton RC, Lee M, Brotzman LE, et al. Use of social network analysis in the development, dissemination, implementation, and sustainability of health behavior interventions for adults: a systematic review. Soc Sci Med 2019;220:81-101.

52 Scott J. Social network analysis: developments, advances, and prospects. Soc Netw Anal Min 2011;1:21-6.

53 Serrat O. Social network analysis. In: knowledge solutions tools methods, and approaches to drive organizational performance. Singapore: Springer, 2017: 39-43.

54 Grol R, Wensing M, Eccles M. Improving patient care: the implementation of change in clinical practice. Edinburgh, Scotland: Elsevier Butterworth Heinemann, 2005.

55 Glasgow RE, McKay HG, Piette JD, et al. The RE-AIM framework for evaluating interventions: what can it tell us about approaches to chronic illness management? Patient Educ Couns 2001;44:119-27.

56 Abraham C, Michie S. A taxonomy of behavior change techniques used in interventions. Health Psychol 2008;27:379-87.

57 Bartholomew L, Parcel G, Kok G, et al. Intervention mapping: designing theory and evidence-based health promotion programs. Mountain View, CA: Mayfield, 2001.

58 van Meijel B, Gamel C, van Swieten-Duijfjes B, et al. The development of evidence-based nursing interventions: methodological considerations. J Adv Nurs 2004;48:84-92.
59 Bleijenberg N, de Man-van Ginkel JM, Trappenburg JCA, et al. Increasing value and reducing waste by optimizing the development of complex interventions: enriching the development phase of the medical research council (MRC) framework. Int J Nurs Stud 2018;79:86-93.

60 Valente TW, Palinkas LA, Czaja S, et al. Social network analysis for program implementation. PLoS One 2015;10:e0131712.

61 Latkin CA, Knowlton AR. Social network assessments and interventions for health behavior change: a critical review. Behav Med 2015;41:90-7.

62 Perkins JM, Subramanian SV, Christakis NA. Social networks and health: a systematic review of sociocentric network studies in lowand middle-income countries. Soc Sci Med 2015;125:60-78.

63 Macro BM. meso, micro: broadening the 'social' of social network analysis with a mixed methods approach. Qual Quant 2016;50:2217-36.

64 Domínguez S, Hollstein B. Mixed methods social networks research: design and applications. New York, NY: Cambridge University Press, 2014.

65 Rienties B, Johan N, Jindal-Snape D. Bridge building potential in cross-cultural learning: a mixed method study. Asia Pacific Educ Rev 2015;16:37-48

66 Schoonenboom J. Designing mixed methods research by mixing and merging methodologies: a 13-step model. Am Behav Sci 2018;62:998-1015.

67 Schoonenboom J, Johnson RB, Froehlich DE, et al. Combining multiple purposes of mixing within a mixed methods research design. Int J Mult Res Approaches 2018;10:271-82.

68 Subirana M, Solá I, Garcia JM, et al. A nursing qualitative systematic review required MEDLINE and CINAHL for study identification. J Clin Epidemiol 2005;58:20-5

69 Hand C, Letts L. Occupational therapy research and practice involving adults with chronic diseases: a scoping review and internet scan. Ottawa: Canadian Association of Occupational Therapists, 2009.

70 Brien SE, Lorenzetti DL, Lewis S, et al. Overview of a formal scoping review on health system report cards. Implement Sci 2010;5:2.

71 Njelesani J, Couto S, Cameron D. Disability and rehabilitation in Tanzania: a review of the literature. Disabil Rehabil 2011;33:2196-207.

72 McColl MA, Shortt S, Godwin M, et al. Models for integrating rehabilitation and primary care: a scoping study. Arch Phys Med Rehabil 2009;90:1523-31. 